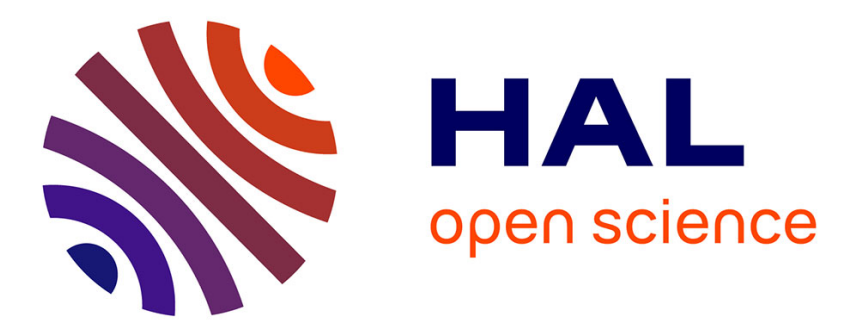

\title{
Origami-based auxetic tunable Helmholtz resonator for noise control
}

Amine Benouhiba, Patrick Rougeot, Nicolas Andreff, Kanty Rabenorosoa, Morvan Ouisse

\section{- To cite this version:}

Amine Benouhiba, Patrick Rougeot, Nicolas Andreff, Kanty Rabenorosoa, Morvan Ouisse. Origamibased auxetic tunable Helmholtz resonator for noise control. Smart Materials and Structures, 2021, 30 (3), pp.35029. hal-03186557

\section{HAL Id: hal-03186557 https://hal.science/hal-03186557}

Submitted on 31 Mar 2021

HAL is a multi-disciplinary open access archive for the deposit and dissemination of scientific research documents, whether they are published or not. The documents may come from teaching and research institutions in France or abroad, or from public or private research centers.
L'archive ouverte pluridisciplinaire HAL, est destinée au dépôt et à la diffusion de documents scientifiques de niveau recherche, publiés ou non, émanant des établissements d'enseignement et de recherche français ou étrangers, des laboratoires publics ou privés. 


\title{
Origami-based auxetic tunable Helmholtz resonator for noise control
}

\author{
Amine Benouhiba, Patrick Rougeot, Nicolas Andreff, Kanty \\ Rabenorosoa, Morvan Ouisse \\ FEMTO-ST Institute, Univ. Bourgogne Franche-Comté,CNRS, Besançon, France \\ E-mail: amine.benouhiba@femto-st.fr, morvan.ouisse@femto-st.fr
}

October 2020

\begin{abstract}
In this paper, we propose an origami-based auxetic tunable Helmholtz resonator. By controlling the volume of the cavity, a real-time tunability of the resonance frequency can be achieved. The design is inspired by the waterbomb origami base, which expands when stretched and contracts when compressed. Such a foldable structure offers a wide range of volume variation, which corresponds to a larger frequency shift in the bandwidth of interest. Based on the design of the origami resonator, multi-physical models are established to understand and predict the parameters' effect on the device's behavior. After fabrication, experimental tests are conducted on physical prototypes to validate the models and illustrate the effectiveness of the concept.
\end{abstract}

Keywords: tunable Helmholtz resonators, origami-based design, acoustics, smart structures, absorption efficiency.

\section{Introduction}

In the old ages, the Greeks and Romans used several arranged cavities in their theater walls to improve the acoustics. Later, the same principle was applied in churches and mosques. In the 1st century BC, they were called "Vitruvian vases", named after the engineer, architect and the author of "de Architectura", which is considered to be the oldest book on acoustics in buildings [1]. However, the resonant cavity theory was introduced for the first time, by Hermann von Helmholtz (1860) then Lord Rayleigh (1870). Moreover, even though these resonators bear the name of Helmholtz, it is Rayleigh's work, presented in his book: "On the theory of resonators" [2], which is best known and studied. If the analogy of the Vitruvian vases with the Helmholtz resonators is made, it is understood that according to the dimensions and the geometrical characteristics of the cavity and/or the neck, the role of the resonator can be very different: amplification or attenuation [1]. There are two distinctive methods to achieve noise reduction in acoustic applications, passive noise control, and active noise control. 
The latter is achieved by introducing a canceling wave $\left(180^{\circ}\right.$ out of phase "antinoise" wave) through an appropriate array of secondary sources [3]. In contrast, passive noise control is achieved by relying on certain materials and structures that have the ability to absorb acoustic energy, such as porous materials and Helmholtz resonators. Although porous materials have a broadband effect, their amplitude is limited, and their effectiveness in low frequencies is directly correlated with the thickness of the material. On the other hand, Helmholtz resonators are very effective in low frequencies, but only in a very narrow bandwidth. As a result, the main advantage of active techniques over passive techniques is the implicit adaptability of the control system to changing excitations [4].

Helmholtz resonators are usually utilized for the control of steady, harmonic sound fields with tonal, and narrowband spectrums. Thanks to their interesting vibroacoustic properties, they have demonstrated widespread usefulness in reverberant environments such as churches [5], mufflers in pipes, and ducts [6], as well as numerous other applications. Their noise reduction ability made them the subject of numerous studies from researchers and engineers. These studies include the theory of generalized Helmholtz resonator [7], properties and modeling of cylindrical Helmholtz resonators [8, 9, 10], effects of Helmholtz resonator's neck [11, 12], and hybrid acoustic systems based on Helmholtz resonator $[13,14,15]$. The main advantages of Helmholtz resonators are their simplicity and efficiency at low frequencies. Nevertheless, their relatively narrow bandwidth represents a significant limitation. Considering that, these resonators need to be precisely tuned to achieve significant noise attenuation, any fluctuation in the incident frequency or the environment conditions instigate a tuning for such devices, as some mistuned resonators can sometimes induce noise rather than reducing it.

Adaptive-passive devices are intelligent systems that can adapt their passive properties to maintain their high performance under changing working conditions. Having the ability to adjust the geometrical properties of Helmholtz resonators, such as the length of the neck or the volume, will allow for accurate, and real-time tuning of the devices in response to the variations of the excitation frequency. Therefore, tunable Helmholtz resonators can be very beneficial. There are many examples in the literature regarding tunable Helmholtz resonators. De Bedout et al. [16] introduced a self-tuning Helmholtz resonator for adaptive-passive noise control, with a varying cavity volume. The volume variation was achieved by adding two rigid walls inside the cylindrical cavity of the resonator: a fixed wall, which was glued to the cylindrical sidewall and the top plate, and a movable wall, which is glued to the bottom plate only; as a result, it can rotate freely. Both walls are linked to each other at the center of the cavity; hence, a rotational movement of the movable wall can be easily achieved. Moreover, the bottom plate is attached to a DC motor, so by rotating the bottom plate, the movable wall rotates also, changing in the process the volume of the resonator cavity. Yu et al. [17] introduced a tunable acoustic metamaterial with an array of resonators actuated by Dielectric Elastomer (DE). The array contains four DE resonators connected in series to a rigid 
duct, each one with a slightly different pre-stretch ratio. The array provides a relatively wide attenuation bandwidth (compared to the use of a single DE resonator), which can be shifted using an applied voltage. Lastly, Abbad et al. [18, 13] presented an adaptive Helmholtz resonator based on DE, embedded in a melamine foam. The proposed concept consists in replacing the top wall of a conventional cylindrical Helmholtz resonator with a dielectric membrane. The stiffness of the membrane, which depends primarily (in the passive state) on the pre-stretch ratio, is controlled via an applied voltage. Also, as the change in the membrane stiffness causes a frequency shift, a tunable Helmholtz resonator using an applied voltage can be achieved. Compared to traditional passive devices, adaptive-passive noise control devices guarantee high performances in the lowfrequency range despite their narrow bandwidth. Moreover, compared to active noise control, they do not require complex control algorithms, and they need minimal power consumption.

In this paper, an origami-based auxetic tunable Helmholtz resonator is proposed. The design, briefly introduced before in [19], is based on the waterbomb origami base. Such a foldable structure offers a wide range of volume variation, which corresponds to a larger frequency shift in the bandwidth of interest. There are many examples of origamibased acoustic devices in the literature, such as ultrasound transmitters [20, 21], noise reduction devices [22], wave guides [23, 24, 25], vibration isolation structures [26], etc. Furthermore, while almost all the mentioned works use numerical simulations to model the acoustic devices' behavior, a good number of them use analytical models as well. Furthermore, tunability, which is inherited from origami-based systems and thus allows for a real-time adjustment of properties, has been a long sought-after ability. Therefore, tunable origami-based devices are not restricted to acoustic devices only; there are other examples, including electromagnetic devices, such as tunable metamaterials $[27,28]$ and self-foldable reflector antennas [29, 30].

In the transport industry, noise and vibration control is a major requirement in order to enable high performance, as well as to ensure customer satisfaction. Against varying tonal excitations, the use of origami Helmholtz resonators can be very beneficial, thanks to their real-time tunability. The targeted, practical applications for such devices are the aeronautic field as well as ducts and ventilation systems.

This paper investigates the acoustic behavior of an innovative tunable Helmholtz resonators, and it does so within the framework of different sections. Section 2 addresses the proposed origami concept and the design of the resonator. Section 3 discusses the two different models for describing the acoustic behavior of origami-based Helmholtz resonators: a P-TMM analytical model based on the concept of an equivalent volume, and a finite element model. Section 4 presents the results and discusses the acoustics performances of the origami-based Helmholtz resonators. Lastly, section 5 concludes the paper and addresses the future work as well as the applications of such acoustic devices. 


\section{Concept}

The analytical model for calculating the natural frequency of a cavity resonator with a circular neck was first developed by Hermann von Helmholtz [31]:

$$
f_{r}=\frac{c_{0}}{2 \pi} \sqrt{\frac{A}{d_{n} V}},
$$

where the variables are described in Tab. 1.

However, the Eqn. (1) does not entirely agree with the experiment. The measured frequency is lower than the predicted value. This was first observed by Lord Rayleigh [32], who proposed an effective neck length, $d_{n}^{\prime}=d_{n}+\delta$, in which a correction was added to the physical length of the neck to account for the effect of the extremities. Several refinements have been made by a number of authors, who also take into account different resonator geometries, including the effect of the extremities: please refer to Ingard et al. [33], Alster et al. [34], Panton et al. [35] and Chanaud et al. [9]. As a results, for a sound wave length $\lambda>>R_{n}$, the effective increase of the neck length at the cavity is approximated by $\frac{8 R_{n}}{3 \pi} \approx 0.85 R_{n}$, and the effective increase of the neck length at the open end by $0.61 R_{n}$, which means that: $d_{n}^{\prime}=d_{n}+1.46 R_{n}$. Thus, Eqn. (1) will be as such:

$$
f_{r}=\frac{c_{0}}{2 \pi} \sqrt{\frac{A}{d_{n}^{\prime} V}} .
$$

Based on this analytical model, the resonant frequency is controlled by the dimensions of the Helmholtz resonator, whether those of the neck, the cavity, or both. Nonetheless, this paper only discusses the variation of the cavity parameters for two reasons:

(i) the neck is relatively much smaller than the cavity, which makes varying its geometrical properties that much more complicated;

(ii) unlike the cavity where the only important property is its size (this is only the case for Helmholtz resonators with cylindrical rigid necks), the shape of the neck in addition to its size has a high influence on the acoustic properties of the resonator, thus trying to study the behavior of a Helmholtz resonator by altering the geometrical properties of its neck is a far more complex process at this stage.

Moreover, according to Fig. 1, which is made using the variables of Tab. 1, it is more efficient to act on the radius of the cavity rather than the length. That said, acting simultaneously on the radius and the length, by employing an auxetic structure, is the most effective approach. Therefore, the proposed design (see Appendix A) is based on the waterbomb origami base, which exhibits an auxetic behavior (negative Poisson's ratio). The waterbomb base has been the focus of several works [36, 37, 38]. It has also been used for the creation of many interesting devices, including an auto-deployable origami stent graft [39], a crawling worm robot [40], and deformable origami wheels [41]. One of the reasons for using the waterbomb base lies in the fact that it can be used to create tubular auxetics. In other words, they display a counter-intuitive behavior, as 
under uniaxial compression (tension), these mechanisms contract (expand) transversely. There are many examples of tubular auxetics in the literature. For instance, Karnessis et al. [42] studied the mechanical behavior of auxetic tubes based on inverted hexagonal honeycombs, while Chen et al. [43] investigated the effects of pattern parameters on tubular auxetics for microcatheters.

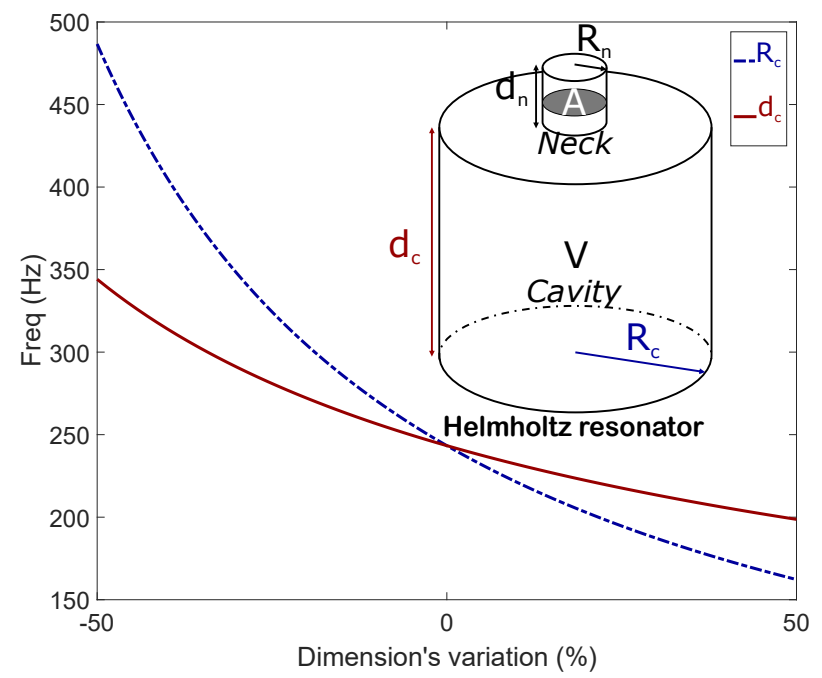

Figure 1. Comparison between the influence of the radius $R_{c}$ and the length of a cylindrical cavity $d_{c}$ of Helmholtz resonator on its theoretical natural frequency.

Table 1. List of variables.

\begin{tabular}{llll}
\hline Variables & Definition & Value & Unit \\
\hline \hline$c_{0}$ & Speed of sound in air & 343 & $\mathrm{~m} \cdot \mathrm{s}^{-1}$ \\
$R_{n}$ & Radius of the neck & 6 & $\mathrm{~mm}$ \\
$d_{n}$ & Depth of the neck & 20 & $\mathrm{~mm}$ \\
$R_{c}$ & Radius of the cavity & 30 & $\mathrm{~mm}$ \\
$d_{c}$ & Depth of the cavity & 70 & $\mathrm{~mm}$ \\
$A$ & Cross-section of the neck & $\pi R_{n}^{2}$ & $\mathrm{~mm}^{2}$ \\
$V$ & Volume of the cavity & $\pi R_{c}^{2} d_{c}$ & $\mathrm{~mm}^{3}$ \\
\hline
\end{tabular}

The full 3D design (i.e., the folded shape) of the origami-based Helmholtz resonator is shown in Fig. 2. The proposed origami design offers many advantages, which include high tunability, low weight, and a minimal number of parts to reduce acoustic leakage. Furthermore, it ensures an entirely closed cavity (fully functional Helmholtz resonator), and a minimal geometrical variation for maximum tunability, thanks to its auxetic property. 


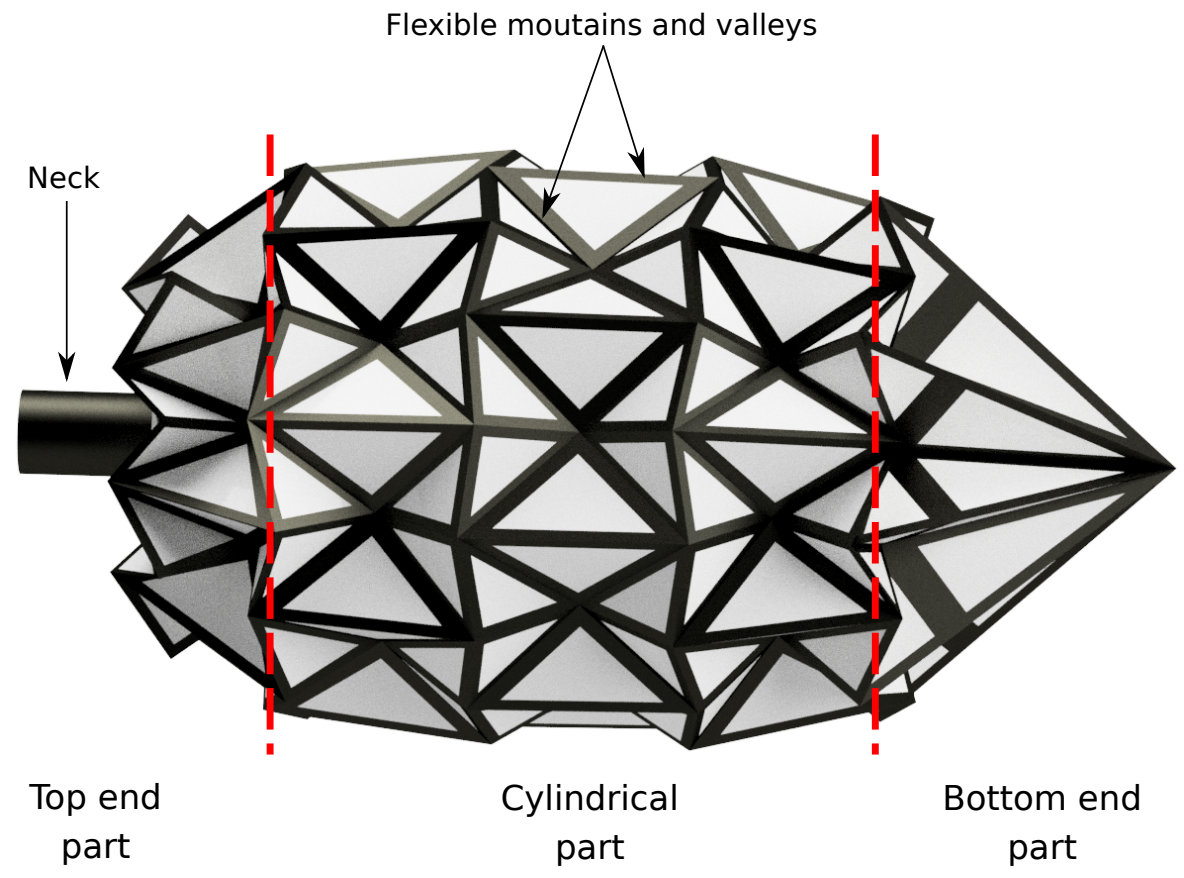

Figure 2. 3D design of the proposed origami Helmholtz resonator.

\section{Modeling}

\subsection{Equivalent volume model}

In this work, the P-TMM is used as a simplified model for the design and investigation of the acoustic behavior of the origami-based acoustic devices. The system is an origamibased Helmholtz resonator, which, for the purpose of this model, can be replaced for each state of folding by a conventional Helmholtz resonator with a cuboid cavity that holds the same volume as the original. As the shape of the cavity in this model is different from the real one, it is expected that some physical phenomena could not be described by this model, which is presented in Appendix B. Such a model was used by Doutres et al. [44], to model the behavior of a hybrid acoustic system, composed of conventional Helmholtz resonators embedded in a homogeneous porous material (foam) in a periodic fashion, which makes for a full spectrum noise control system, foam for high and medium frequencies, and Helmholtz resonator for low frequencies. A finite element model will be presented later to illustrate the efficiency of the P-TMM approach, as an efficient tool for the design of adaptive Helmholtz resonators.

\subsection{Finite element Model}

A numerical model capable of describing the acoustic behavior of Helmholtz resonators with arbitrarily complex shapes, including origami designs, was developed. The numerical model can be used to validate the equivalent volume model discussed above in the case of a 1D acoustic excitation. The former reproduces the transmission tube tests (see Fig. 3). The acoustic fields inside the tube and the resonators cavities are described by Helmholtz equation, and the details of the used model can be found in 
Tab. 2 .

Table 2. Details of the finite element model.

\begin{tabular}{ll}
\hline Software & Comsol Multiphysics 5.3A \\
Type element & Free tetrahedral \\
Maximum element size & $c_{0} /\left(6 \times{ }^{\dagger} f_{\text {max }}\right)$ \\
Mesh convergence & 10437 element \\
Solver & MUltifrontal Massively Parallel Solver (MUMPS) \\
\hline
\end{tabular}

${ }^{\dagger} f_{\text {max }}$ is the highest used frequency.

To simulate the normal incidence excitation, a unit pressure is applied from one side of the tube, while the other side is considered to be acoustically rigid. Using the resulted virtual measurements of the acoustic pressure at two different locations, which serve as microphone locations, the normal acoustic absorption was deduced according to the one load, two microphone technique [45].

The modeled acoustic devices are origami-based Helmholtz resonators with circular neck aperture. The walls of the resonators are modeled as impermeable, rigid, and motionless. Moreover, The Johnson-Champoux-Allard equivalent fluid rigid model is used to account for viscous and thermal dissipations caused by the resonator's neck. The effective properties of the air layer of the neck were determined by a cylindrical pore model [46]. The values of the parameters of the model are provided in Tab. 3. The numerical simulations will be compared to the natural frequency model, equivalent volume model (P-TMM model), and experimental tests conducted in a $100 \mathrm{~mm}$ cylindrical acoustic transmission tube. The simulations are carried out for Helmholtz resonators in the $\mathrm{AB}$ and $\mathrm{BA}$ positions. Furthermore, since both the acoustic

Table 3. Parameters of the Johnson-Champoux-Allard equivalent fluid model used for the neck.

\begin{tabular}{lllll}
\hline Variables & Definition & Expression [47] & Value & Unit \\
\hline \hline$\phi$ & Porosity & - & 1 & - \\
$\sigma$ & Airflow resistivity & $\frac{8 \eta}{R_{n}^{2}}$ & 4 & $\mathrm{Nsm}$ \\
$\alpha_{\infty}$ & Tortuosity & - & 1 & - \\
$\Lambda$ & Viscous characteristic length & $R_{n}$ & 6 & $\mathrm{~mm}$ \\
$\Lambda^{\prime}$ & Thermal characteristic length & $R_{n}$ & 6 & $\mathrm{~mm}$ \\
$\rho_{0}$ & Density & - & 1.2 & $\mathrm{kgm}^{-3}$ \\
\hline
\end{tabular}



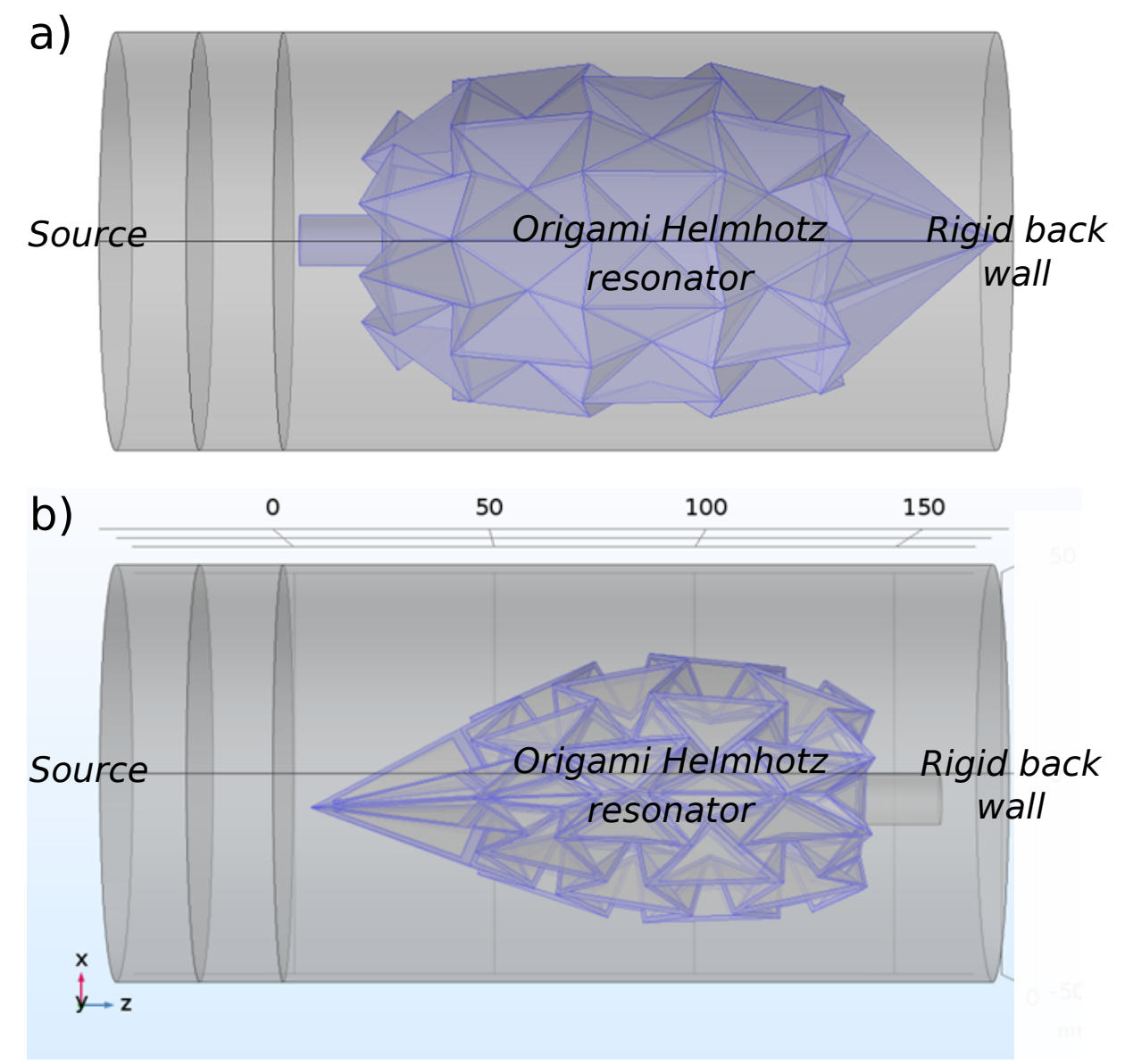

Figure 3. One load, two microphone technique, where (a) the origami-based Helmholtz resonator (unfolded state) is placed inside the transmission tube in $\mathrm{AB}$ position, and (b) the origami-based Helmholtz resonator (folded state) is placed inside the transmission tube in BA position.

transmission tube and the neck of the resonator are cylindrical, and the origami-based cavity of the resonator is also symmetrical, by considering symmetry only an $8^{\text {th }}$ of the acoustic device can be modeled for plane wave excitation. This will decrease the computation time significantly, compared to the full model.

\section{Results and discussion}

To better understand the behavior of such devices, different prototypes with different properties and purposes were fabricated. As a result, it was possible to isolate the different properties and evaluate them separately. Two types of origami-based Helmholtz resonators were fabricated: 3D printed rigid origami-based Helmholtz resonators, each with a different folding state (folded, semi-folded and unfolded), and a tunable origamibased resonator, as shown in Fig. 4 (more details about the fabrication can be found in Appendix C). The study, characterization, and modeling of these origami-based structures can help to better understand and quantify their acoustic properties, in 


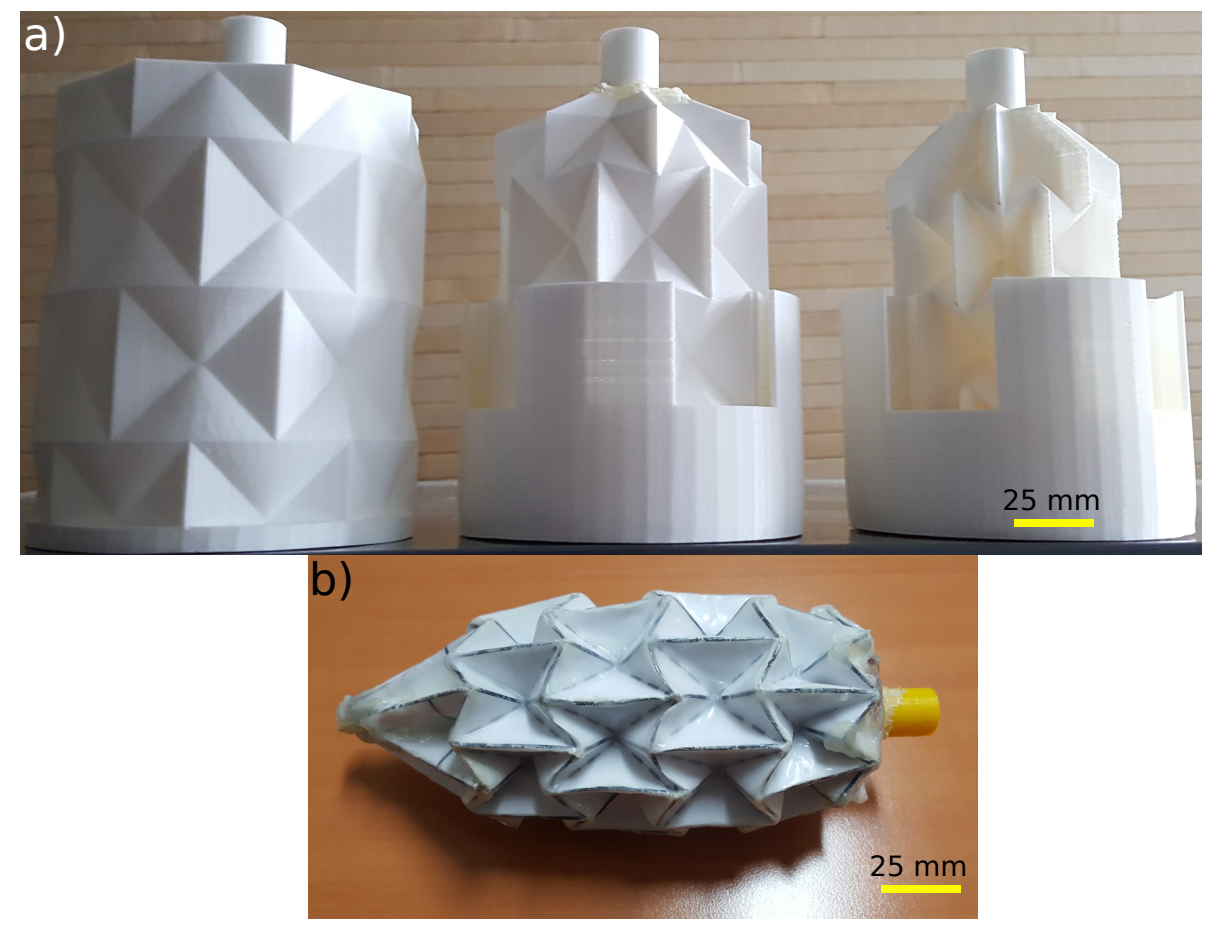

Figure 4. Different types of the fabricated origami-based Helmholtz resonators: a) 3D printed rigid origami-based resonators, unfolded, semi-folded, and folded form left to right, respectively, and b) tunable origami-based resonator.

particular, as well as improve the process of designing high-performance origami-based acoustic devices, in general.

\subsection{Rigid origami-based Helmholtz resonator}

In this section, experimental testing on origami-based rigid Helmholtz resonators is conducted. Three rigid resonators with different states of folding (unfolded, semi-folded, and folded) were 3D printed. The size and the folding states of the origami-based Helmholtz resonator were arbitrarily selected. Using an acoustic transmission tube, a one-load two-microphone technique [45] was used to evaluate the acoustic absorption of three resonators. The latter were inserted in a forward position (in the the AB position as shown in Fig. 3) without leaving any space between the back of the resonators and the end wall of the tube. No foam was added with the resonators; instead, supports were printed to keep the resonators well centered inside the transmission tube, especially for the semi-folded and the folded resonators.

The measured behaviors of the three rigid origami-based resonators are similar to those of a conventional Helmholtz resonator with a cylindrical neck and cavity, a very high acoustic absorption in the low-frequency range $(50-500 \mathrm{~Hz})$ with a narrow (very selective) bandwidth, as shown in Fig. 5 and Tab. 4. As all tests were performed in the same 100 mm-diameter tube, it is not surprising to see the folded resonator achieve the lowest absorption rate. The folded origami-based resonator has a smaller diameter; therefore, it occupies less of the testing tube's cross-section, which leads to some acoustic leakage compared to the remaining two resonators. However, we assume that the imperfect absorption of the unfolded resonator (93\%) is due to improper fabrication (3D printing defects) rather than anything else. The relationship between the different absorption 

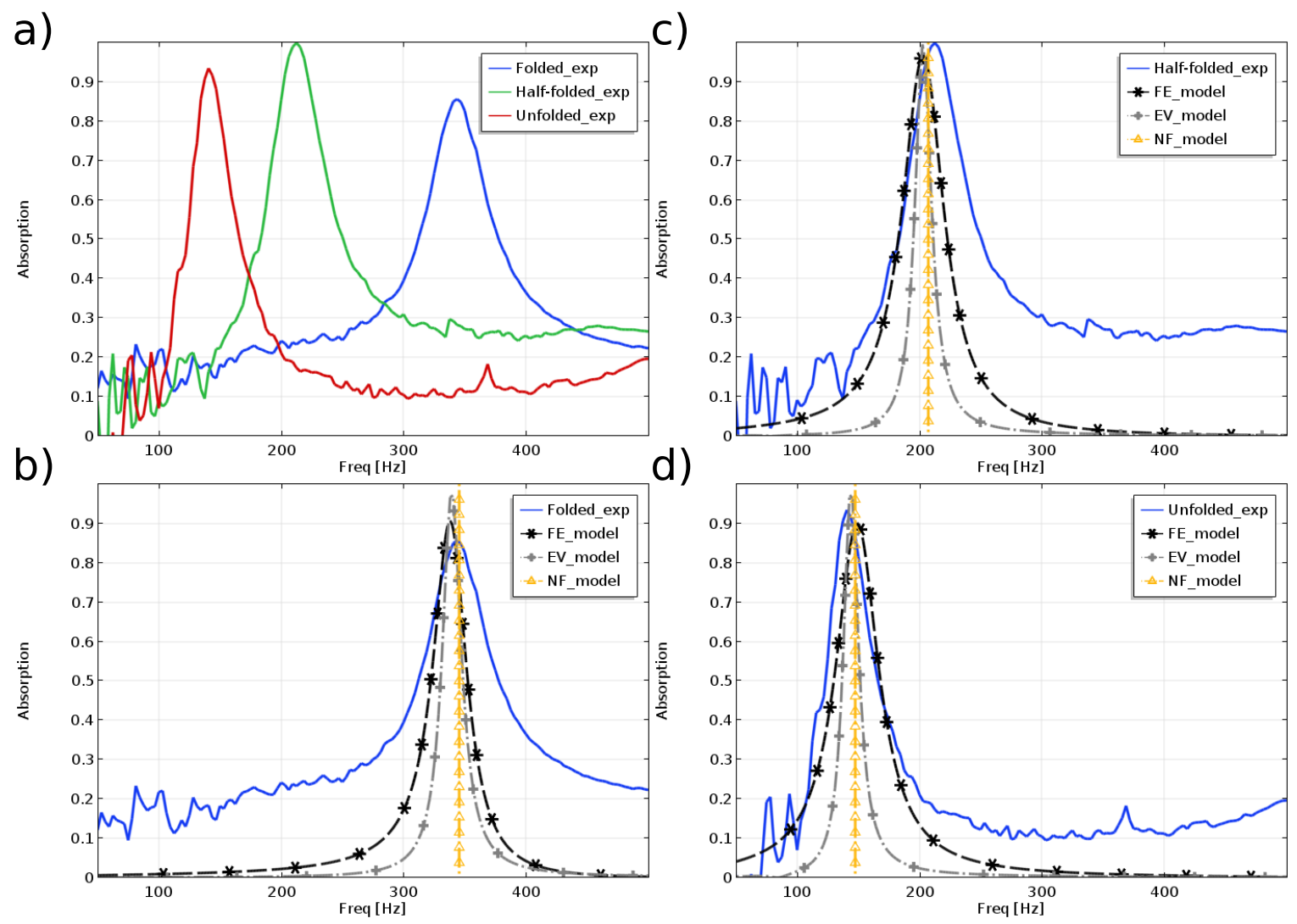

Figure 5. Comparison between the a) experimental results of the acoustic performance (acoustic absorption) of 3D printed rigid origami Helmholtz resonators and the FE model, the equivalent volume (EV) model, and the natural frequency (NF) model, for three different states of folding: b) folded $(340 \mathrm{~Hz})$, c) half-folded $(212 \mathrm{~Hz})$, and d) unfolded $(140 \mathrm{~Hz})$.

peaks is also consistent with that of conventional Helmholtz resonators: the value of the resonance frequency given by Eqn. 2, referred to as NF (for Natural Frequency) in Fig. 5, is perfectly in accordance with the measurements. These results are also compared to those obtained with the FE model (see Sec. 3.2). The latter uses a Johnson-ChampouxAllard model to simulate the thermo-viscous losses produced by the neck. The model also considers all the walls of the cavity and the neck to be acoustically rigid without having any mechanical properties.

Furthermore, these results are also in a good agreement with the analytical model referred to as EV (for Equivalent Volume, see Sec. 3.1) model, which, in addition to the $\mathrm{NF}$, both were used to design the system. As a result, since the analytical models do not take into account the shape of the cavity, it is safe to assume that the shape of the cavity (origami shape) has no influence on the resonators acoustic performance, at least with regard to the resonance frequency.

To summarize, the NF model is able to estimate the resonance frequency of the origamibased Helmholtz resonator. Additionally, the EV model, which is validated by the FE model, is a fast analytical tool for the design of origami-based Helmholtz resonators, as it is able to provide the frequency evolution of the acoustic performances around the resonance frequency. Lastly, all the developed models are not able to predict the increase in the absorption rate $(\alpha)$ measured in the higher frequency range (see Fig. 5), which may be due to structural behavior or losses in the vicinity of the origami walls, 
as they are known to have an impact on the performances of the resonator [48, 49].

Table 4. Experimental absorption of the rigid origami-based Helmholtz resonators and for different states of folding.

\begin{tabular}{|l||c|c|c|}
\hline State of folding & Unfolded & Semi-folded & Folded \\
\hline \hline Freq. at the peak (Hz) & 140 & 212 & 340 \\
\hline Absorp. At the peak (\%) & 93 & 100 & 86 \\
\hline Average absorption (\%) & \multicolumn{3}{|c|}{93} \\
\hline
\end{tabular}

\subsection{Tunable origami-based resonators}

In the previous section, it has been shown that rigid origami-based Helmholtz resonators act as conventional Helmholtz resonators (for the case of cylindrical necks only). The aim of this section is to investigate the influence of foldability on the acoustic performances to provide tunability, which is the main purpose of using origami design. To that end, a tunable origami-based resonator was fabricated. For the purpose of obtaining such characteristics, the folding lines of the origami cavity need to be almost as rigid as the cavity facets. This gives the resonator acoustic properties comparable to those of a rigid resonator but also makes it harder to fold. However, due to the shape of the folding lines (V-shaped), when forces are applied on the origami cavity, they tend to attract a high concentration of stress, resulting in their deformation; thus, the folding/unfolding is achieved.

Once the tunable resonator was fabricated, a series of acoustic characterization tests were conducted using an acoustic transmission tube. Similar to the rigid resonators, here also a one-load two-microphone technique [45] was utilized to evaluate the acoustic absorption of the resonator. However, in this case, the resonator was inserted in a backward position (in the BA position as shown in Fig. 6) with a $10 \mathrm{~mm}$ space between the resonator and the end wall of the tube. This configuration generates a better performance in terms of the acoustic absorption rate. The resonator is folded into different diameters and tested each time. Rings with diameters ranging from 70 to $50 \mathrm{~mm}$ are utilized to adjust the middle section of the origami cavity to the desired folding state. The experimental results are as expected, high acoustic absorption peaks in the low-frequency range $(50-500 \mathrm{~Hz})$ with a narrow (very selective) bandwidth. It is observed that the bigger the cavity volume is, the lower is the corresponding resonance frequency (as shown in Fig. 7). Acoustic properties exhibited by the tunable origamibased resonator are very consistent with those of a rigid origami-based resonator, which makes them also consistent with a conventional Helmholtz resonator as well. It is worth noting, however, even though the absorption rate of the tunable resonator is high, with an average above the $80 \%$ for the five tested folding states, it is still slightly lower than the rate of a rigid Helmholtz resonator (origami or otherwise). Nonetheless, it is believed that the absorption rate of the tunable resonator can be improved using better fabrication materials and high-performance manufacturing machines. The tunable resonator is able to generate a frequency shift of $81 \mathrm{~Hz}$ in the low end of the lowfrequency domain, starting from $138 \mathrm{~Hz}$ (diameter of $70 \mathrm{~mm}$ ) to $219 \mathrm{~Hz}$ (diameter of $50 \mathrm{~mm}$ ). More details are available in Tab. 5. This shows that the tunable origamibased resonator has an impressive ratio of frequency shift to geometrical variation, which averages for more than $4 \mathrm{~Hz}$ for each $1 \mathrm{~mm}$ variation in the diameter of the origami cavity.

For the tunable origami-based Helmholtz resonator, comparing the experimental results 


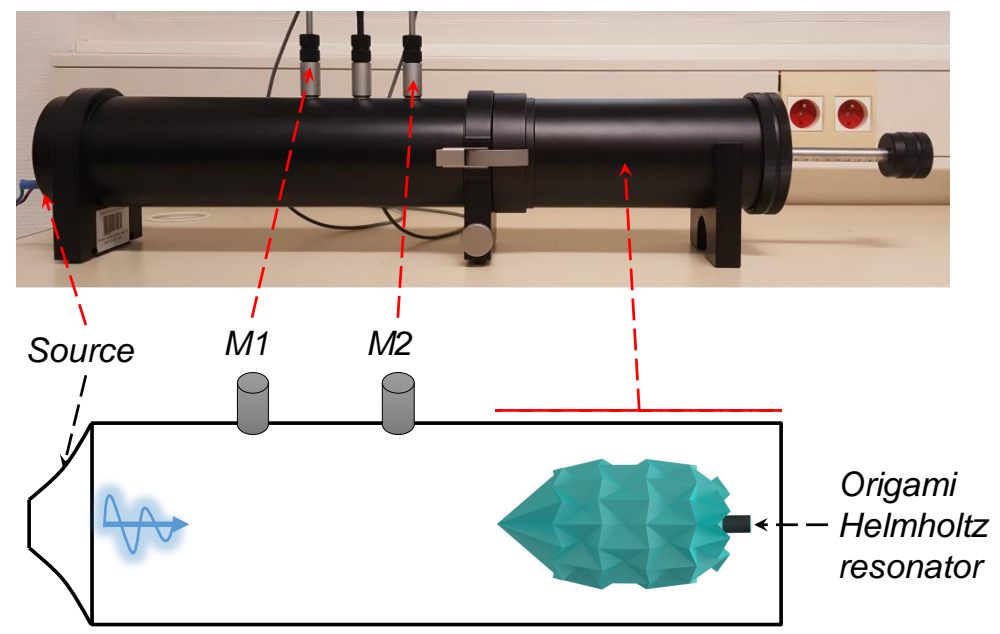

Figure 6. Experimental setup of the one load, two microphone technique, where the origami-based Helmholtz resonator is placed inside the transmission tube in BA position. The transmission tube contains a sound source (loudspeakers) at one end, while two microphones, referred to as "M1", "M2" are used to act as probes, and measure the acoustic pressure.

to the analytical model of the natural frequency to better confirm its acoustic behavior is not possible, as the tested configuration, BA position (backward insertion of the resonator and a $10 \mathrm{~mm}$ space between the latter and the end wall of the tube), cannot be taken into account by a fairly simple model. However, the equivalent volume model (see Sec. 3.1) and FE model (see Sec. 3.2) used above for the rigid resonators can be used here, with the appropriate boundary conditions, and the results are presented in Fig. 7. The good agreement displayed between the experimental results and the models illustrates that the tunable origami-based resonator has an acoustic behavior similar to that of a rigid origami-based as well as a conventional Helmholtz resonator.

Finally, the results highlighted here validate the capabilities of the proposed origami design for a tunable Helmholtz resonator. It is able to achieve using the same origamibased device a wide frequency shift of $81 \mathrm{~Hz}(138-219 \mathrm{~Hz})$ with a relatively low geometrical variation, corresponding to a $20 \mathrm{~mm}$ variation in diameter.

Table 5. Experimental absorption of the tunable origami-based Helmholtz resonator for folding different diameters.

\begin{tabular}{|l||c|c|c|c|c|}
\hline Diameter of the origami HR $(\mathbf{m m})$ & $\mathbf{7 0}$ & $\mathbf{6 5}$ & $\mathbf{6 0}$ & $\mathbf{5 5}$ & $\mathbf{5 0}$ \\
\hline \hline Freq. at the peak (Hz) & 138 & 150 & 167 & 188 & 219 \\
\hline Absorp. At the peak (\%) & 81 & 80 & 83 & 76 & 81 \\
\hline Total bandwidth (Hz) & \multicolumn{5}{|c|}{$81(59 \%)^{*}$} \\
\hline Average absorption (\%) & \multicolumn{5}{|c|}{80.2} \\
\hline
\end{tabular}

*Relative bandwidth compared to its low frequency $(138 \mathrm{~Hz})$ 

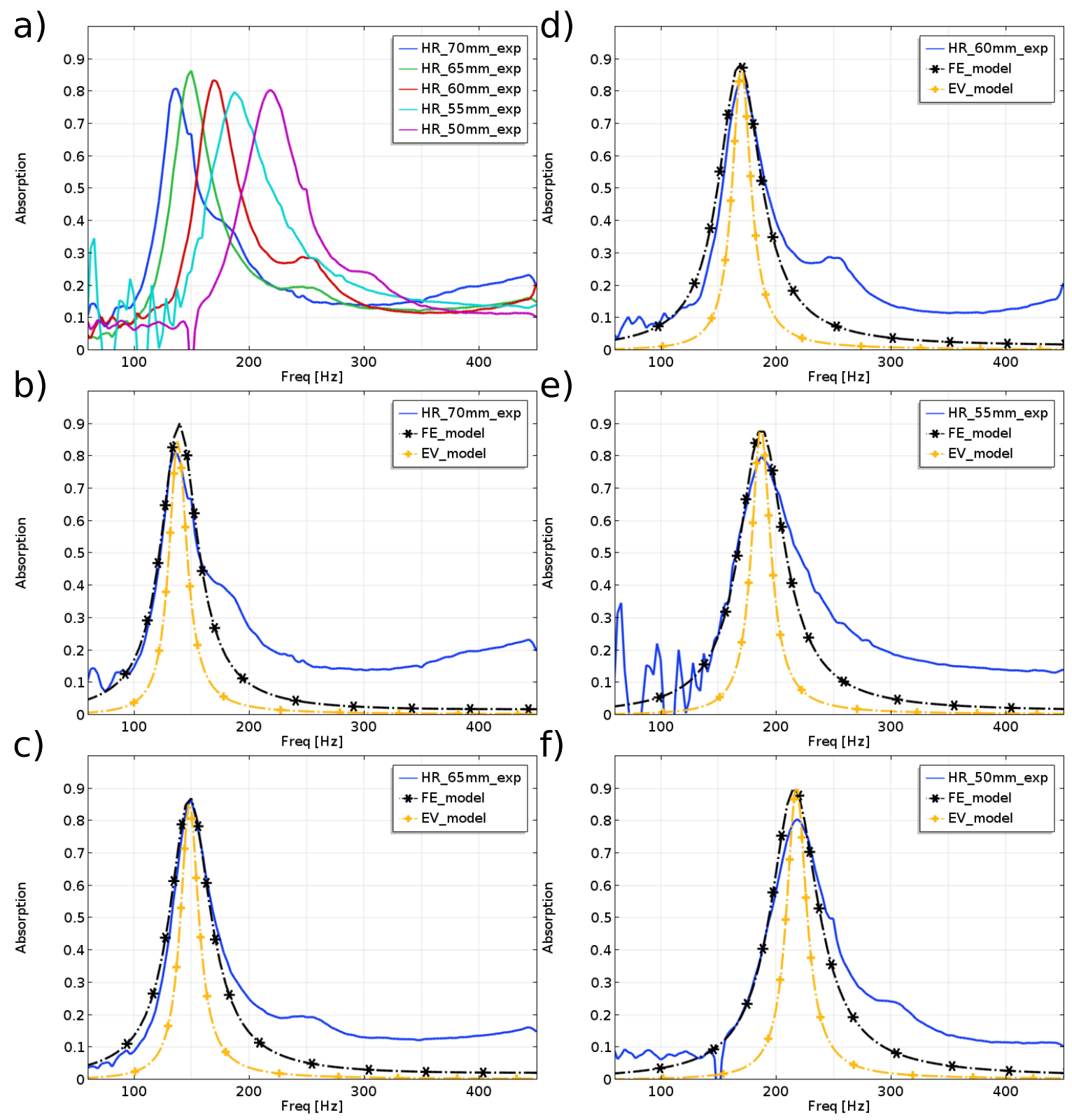

Figure 7. Comparison between the a) experimental results of the acoustic performance (acoustic absorption) of a tunable origami-based Helmholtz resonator and the FE model, and the equivalent volume (EV) model, for different values of the cavity diameter: b) $70(138 \mathrm{~Hz})$, c) $65(150 \mathrm{~Hz})$, d) $60(167 \mathrm{~Hz})$, e) $55(188 \mathrm{~Hz})$, and f) $50 \mathrm{~mm}(219 \mathrm{~Hz})$.

\section{Conclusion}

Helmholtz resonators are simple yet effective low-frequency sound control devices. As a result, they attracted the attention of many engineers and scientists as a promising concept for vibro-acoustic applications. In this work, due to the need for adaptive-passive noise control, an origami-based auxetic tunable Helmholtz resonator was proposed, modeled, fabricated, and experimentally evaluated. The origami-based 
resonators were modeled using the P-TMM analytical model, based on the equivalent volume concept, as well as, an FE model. Two different Helmholtz resonators were evaluated here, rigid, and tunable origami-based resonators. The latter were able to display fascinating acoustic properties.

During the experimental validation, it was possible to show, that both the rigid and tunable origami-based Helmholtz resonators behave as tradition Helmholtz resonators with cylindrical necks. Moreover, using the tunable origami-based resonator, the capabilities and effectiveness of the auxetic origami design were illustrated. The resonator was able to perform a high frequency shift in the low frequencies of $81 \mathrm{~Hz}$, from 138 to $219 \mathrm{~Hz}$. The frequency shift, which has an average absorption of 80\%, was achieved with a diameter variation of only $20 \mathrm{~mm}$, form $70 \mathrm{~mm}$ to $50 \mathrm{~mm}$. This proves that using the origami design, it is possible to obtain a Helmholtz resonator with very high tunability, and for minimal geometrical variation.

It is worth mentioning that the relatively large absorption observed for high frequencies in the experimental tests is not considered in this work. The absorption is due to structural vibrations of the structure and losses in the constrictions of the origami, and is not Helmholtz resonator related (not caused by the oscillation of the mass of air in the neck); hence, the clear disagreement between the models (analytical and numerical models) and the tests in this region. For these models to take into account such absorption, they should be enriched to include the origami structure's mechanical behavior and thermal and viscous losses in the vicinity of the origami walls. Such work is still undergoing, and more investigating is needed. Additionally, the effect of adding tunability (flexibility), by using origami designs, and their impact on the acoustic properties are still under study and will be included in future works. However, the tools provided here have shown to be very efficient for the design of the resonator in the frequency range of the Helmholtz resonance.

For future applications, it will be interesting to study and investigate the combination of several tunable origami-based Helmholtz resonators with different yet fairly close frequencies, for a broad bandwidth sound control. Moreover, future efforts are also dedicated to the study and analysis of the adapted actuation system as well as the control of such devices. These actuation systems will probably be made from smart materials such as shape memory alloys [50] or polymers [51].

Finally, investigations on the materials to be used for the manufacturing of the origami are required, as the structural behavior is expected to play an important role in the dynamics of the system, and combining Helmholtz resonances with structural resonances could be a promising way to enhance the performances of the acoustic device [13].

\section{Acknowledgment}

This work has been supported by EUR EIPHI (Contract No. ANR-17-EURE-0002), and OrigaBot (Contract No. ANR-18-CE33-0008-03). 


\section{Nomenclature}

$f_{r} \quad$ Natural frequency of Helmholtz resonator.

$c_{0} \quad$ Speed of sound in the fluid (usually air)

$\lambda \quad$ Sound wave length.

$R_{n} \quad$ Radius of neck of the resonator.

$d_{n} \quad$ Length of the neck of the resonator.

$d_{n}^{\prime} \quad$ Effective length of the neck of the resonator.

$A$ Cross-section of the neck of the resonator.

$\delta \quad$ Both exterior and interior ends correction.

$R_{c} \quad$ Radius of the cavity of the resonator.

$d_{c} \quad$ Length of the cavity of the resonator.

$V \quad$ Volume of the cavity of the resonator.

$\phi \quad$ Porosity

$\sigma \quad$ Airflow resistivity

$\alpha_{\infty} \quad$ Tortuosity

$\Lambda \quad$ Viscous characteristic length

$\Lambda^{\prime} \quad$ Thermal characteristic length

$\rho_{0} \quad$ Density

$\mathbf{T}^{n} \quad$ Transfer matrix of an $\mathrm{n}$ element

$\mathbf{Y}^{n} \quad$ Admittance matrix of an $\mathrm{n}$ element

$r_{n} \quad$ Surface ratio of of an $\mathrm{n}$ element

$\rho_{0} \quad$ Air density

$c_{0} \quad$ Speed of sound

$k_{0} \quad$ Wavenumber

$Z_{0} \quad$ Characteristic impedance of air

$M_{s} \quad$ Mass per unit area of the resonator array

$Z_{A} \quad$ Acoustic input impedance

$\eta \quad$ Dynamic fluid viscosity

$R_{s} \quad$ Surface resistance

$s_{i} \quad$ Ratio of the cross-section area of the neck $A$

to the cross-section area of the "internal" elementary cell

$s_{e} \quad$ Ratio of the cross-section area of the neck $A$

to the cross-section area of the "external" elementary cell

$T L_{n} \quad$ Normal incidence sound transmission coefficient

$\tau \quad$ Sound transmission coefficient

$P_{t} \quad$ Transmitted acoustic power

$P_{i} \quad$ Incident acoustic power

$\alpha \quad$ Sound absorption coefficient

$R^{b} \quad$ Sound reflection coefficient 


\section{Appendix A design}

The proposed origami design is based on the well-known origami base, waterbomb (Fig. 8), which exhibits an auxetic behavior. This property allows for a higher volume change, which results in a wider frequency shift in the application of interest. The proposed full origami design of the Helmholtz resonator is divided into three different parts:

(i) a top-end part (Fig. 9.A) which allows the fixation of the neck thereafter. This concept is adapted from Lee et al. [41], and it provides a rigid opening (i.e., with constant geometrical properties) even during the folding and unfolding of the device;

(ii) a middle part (Fig. 9.B), which represents the cavity with the auxetic property;

(iii) and a bottom-end part (Fig. 9.C), which was inspired by the mechanism of the umbrella, as it is required for a Helmholtz resonator to have a closed cavity. Therefore, by using such a design, the cavity can remain perfectly closed during folding and unfolding.

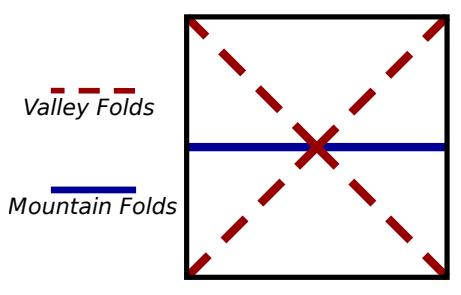

(A)

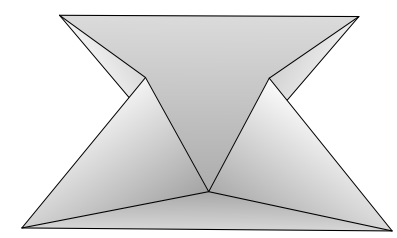

(B)

Figure 8. Waterbomb origami base, the blue lines for the mountain folds and the red ones for the valley folds: (A) flat and (B) folded configurations. 


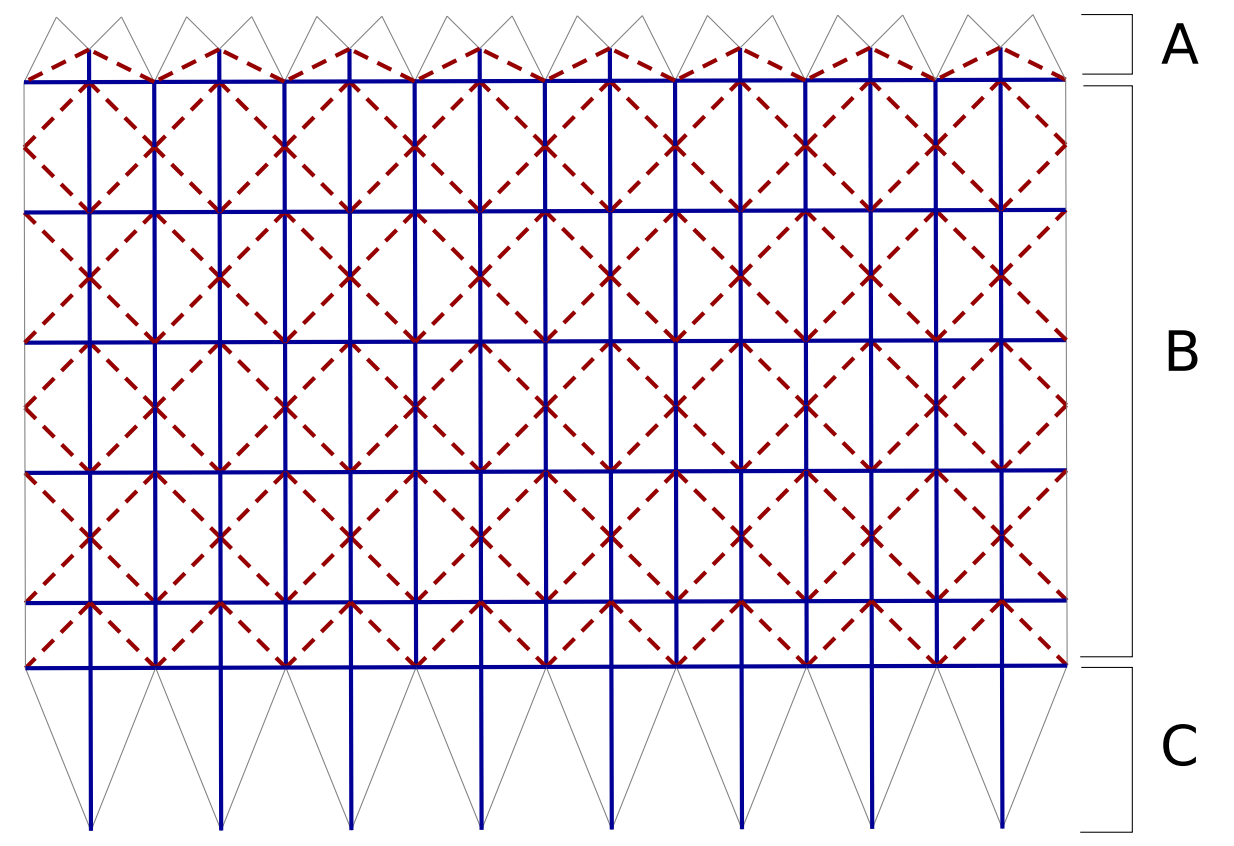

Figure 9. 2D design of the origami-based helmholtz resonator made using the Oripa software [52]: (A) top end part, (B) cylindrical part, and (C) bottom end part. 


\section{Appendix B Equivalent volume model}

In this work, a Transfer Matrix Method (TMM) is used to describe the behavior of tunable Helmholtz resonators. The former is a fast analytical calculation method for the prediction of acoustic performances, in particular for the estimation of the absorption coefficient and the transmission loss of a multilayer composed of several laterally infinite homogeneous layers assembled in series to form a stack. The method is valid under the assumption of a plane wave. This method is expressed by a matrix relation between two acoustic state variables. More or less complex structures, such as single or double walls are usually modeled by the transfer matrix (TMM) method [53]. Recently, Verdière et al. $[54,55]$ proposed an extension of the TMM called the Parallel-TMM approach (PTMM). Consequently, it is possible to take into account both materials in parallel and in series. This method, therefore, makes it possible to model resonant inclusions [44], such as Helmholtz resonators.

\section{P-TMM model}

The acoustic system is defined as a 2D arrangement of an origami cell (OC), which for the purpose of this model is considered to be a cuboid cell, as shown in Fig. 10. Also, the OC is considered to be submerged in a fluid (in this case air). The P-TMM $[54,55,46,56]$ is used to model its 1D acoustical behavior. Furthermore, the OC is considered as a pile of two different layers stacked is series, with thicknesses of $l_{1}$ and $l_{2}$. The first layer contains a parallel assembly of the Helmholtz resonator and an air layer (see Fig. 10), while the second layer contains only air. The transfer matrix of the OC illustrated in Fig. 10 is given by

$$
\mathbf{T}^{O C}=\left[\begin{array}{ll}
T_{11}^{O C} & T_{12}^{O C} \\
T_{21}^{O C} & T_{22}^{O C}
\end{array}\right]=\mathbf{T}^{\text {layer }, 1} \times \mathbf{T}^{\text {layer }, 2} .
$$

Moreover, the transfer matrices of the second layer, the air layer is equal to

$$
\mathbf{T}^{\text {layer }, 2}=\left[\begin{array}{cc}
T_{11}^{2} & T_{12}^{2} \\
T_{21}^{2} & T_{22}^{2}
\end{array}\right]=\left[\begin{array}{cc}
\cos \left(k_{0} l_{2}\right) & j Z_{0} \sin \left(k_{0} l_{2}\right) \\
j \sin \left(k_{0} l_{2}\right) / Z_{0} & \cos \left(k_{0} l_{2}\right)
\end{array}\right]
$$

where $j^{2}=-1, \rho_{0}$ the air density, $c_{0}$ the speed of sound, $k_{0}=\omega / c_{0}$ the wavenumber, and $Z_{0}=\rho_{0} c_{0}$ the characteristic impedance of air. The transfer matrix of the first layer combines two submatrices, which are modeled using a parallel assembly as proposed by Verdière et al [54]. To use P-TMM a number of conditions must be met: (1) only plane waves propagate upstream and downstream of the periodic construction; (2) only normal incidence plane waves propagate in the construction; (3) no pressure diffusion exists between adjacent parallel elements, (4) the wavelength is much larger than the $\mathrm{OC}$, and (5) each element can be represented by a $2 \times 2$ transfer matrix. Conditions (2) to (5) are satisfied here, as we consider (i) low frequencies (i.e., frequencies much lower than $\frac{c_{0}}{L}$ ), (ii) equivalent fluid models for predicting their acoustic behavior and lastly, (iii) a surface impedance model to represent the HR. However, assumption (1) 
seems physically unrealistic around the HR resonance frequency in the vicinity of the HR neck. According to [54], the transfer matrix of layer 1 can be formulated as follows

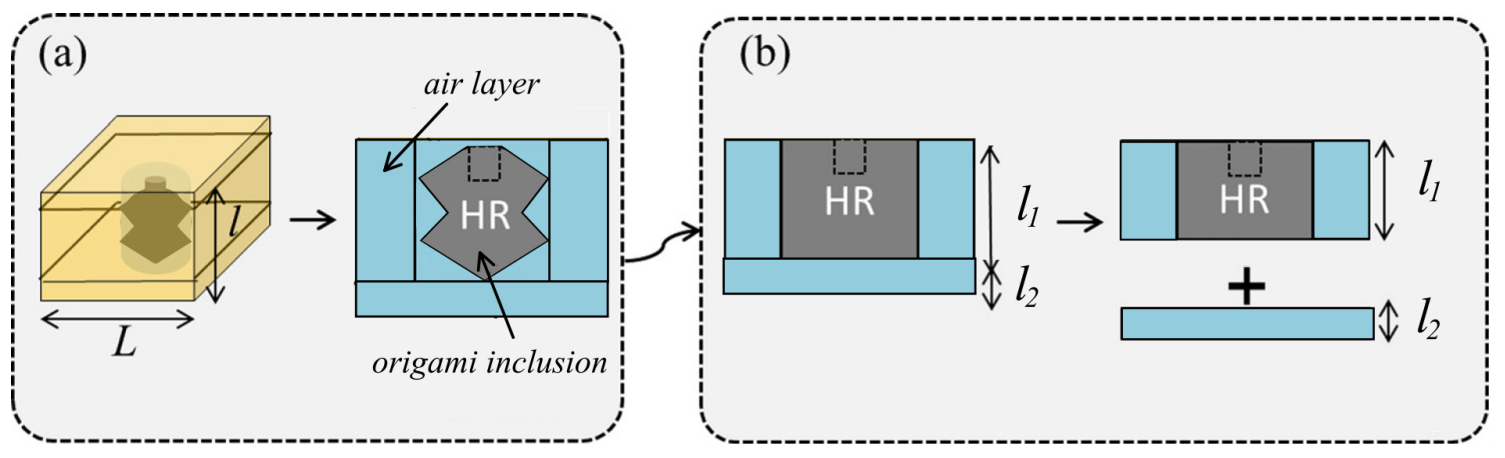

Figure 10. Scheme of the OC with the air layers and the origami-based Helmholtz resonator: (a) illustration of the origami cell and its volume equivalent (b) cuboid cell. (adapted from [44]).

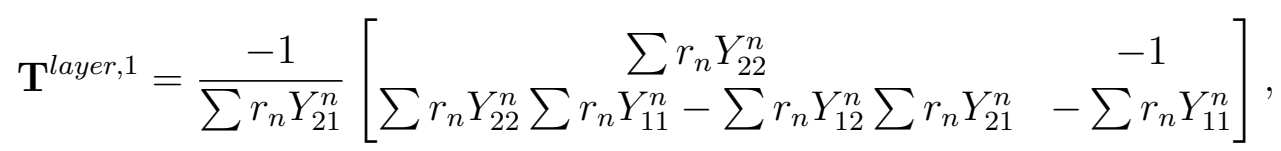

with $\mathbf{Y}^{n}$ the components of the admittance matrix for each element; here $n=$ air for the air layer and $n=H R$ for the resonator element. $r_{n}$ is the surface ratio of each element. Knowing the surface of the origami cell $S_{O C}$ and the surface of the resonator $S_{i}$, thus $r_{H R}=r=S_{i} / S_{O C}$ and $r_{a i r}=1-r_{H R}$. The admittance matrix for each element is given by

$$
\mathbf{Y}^{n}=\left[\begin{array}{cc}
Y_{11}^{n} & Y_{12}^{n} \\
Y_{21}^{n} & Y_{22}^{n}
\end{array}\right]=\frac{1}{T_{12}^{n}}\left[\begin{array}{cc}
T_{22}^{n} & T_{21}^{n} T_{12}^{n}-T_{22}^{n} T_{11}^{n} \\
1 & -T_{11}^{n}
\end{array}\right]
$$

The transfer matrix of the air layer $\mathbf{T}^{\text {air }}$ is given by Eqn. 4 and the one of the resonator $\mathbf{T}^{H R}$ is given in the following section.

\section{Helmholtz resonator model}

As described by [46] and [57], the transfer matrix of the resonator $\mathbf{T}^{H R}$ needed for parallel assemblies with resonant inclusion (represented by Eqn. 5), is written as a product of inertial and acoustic components [57].

The transfer matrix of a Helmholtz resonator positioned so that its neck is facing the acoustic source (this position is called $\mathrm{AB}$ ) is written as

$$
\mathbf{T}^{H R, A B}=\left[\begin{array}{cc}
1 & 0 \\
1 / Z_{A} & 1
\end{array}\right]\left[\begin{array}{cc}
1 & j \omega M_{s} \\
0 & 1
\end{array}\right]
$$

with $M_{s}$ the mass per unit area of the resonator array and $Z_{A}$ the acoustic input impedance. According to [46], the input total impedance of the resonator array under 
normal incidence plane waves, which corresponds to Fig. 10.d, is given by

$$
Z_{A}=\frac{1}{s_{e}}\left(Z_{n e c k}+Z_{0,0}\left(B^{\prime}\right)\right)=\frac{1}{s_{e}}\left(\left[\left(\frac{2 d_{n}}{R_{n}}+4\right) R_{s}+j \omega\left(\varepsilon_{i}+\varepsilon_{e}+d_{n}\right) \rho_{0}\right]-j s_{i} Z_{0} \cot \left(k_{0} d_{c}\right)\right),
$$

where $d_{n}, d_{c}$, and $R_{n}$ are the neck's depth, the cavity depth and the neck's radius, respectively. Additionally, $\eta$ is the dynamic fluid viscosity, $R_{s}$ is the surface resistance: $R_{s}=\left(2 \eta \rho_{0}\right)^{1 / 2} / 2$, and $s_{i}$ and $s_{e}$ are the ratios of the cross-section area of the neck $A$ to the cross-section area of the "internal" and "external" elementary cell, respectively.

The thickness of the walls of the studied resonator is relatively thin; therefore, for the purpose of this model, it is considered negligible. As a result, both cross-section areas, internal and external, have the same value, and it is equal to: $s_{i}=s_{e}=S n / D_{i}^{2}$, where $D_{i}$ is presented in Fig. 10.d. In accordance with the large-wavelength assumption (i.e., $\lambda \gg D i$ and $\left.\lambda \gg d_{c}\right), Z_{0,0}\left(B^{\prime}\right)$ the normal surface impedance of the air layer at the $B^{\prime}$ point is: $Z_{0,0}\left(B^{\prime}\right)=-j s_{i} Z_{0} \cot \left(k_{0} d_{c}\right)$. Moreover, An added length effect (i.e., $\left.\varepsilon_{i}\right)$ is attributed to the resonator's neck depth, to account for the modes of higher-order in the resonator cavity. As mentioned above, the resonator is characterized by the volume of its cavity. Another correction, which is also expressed by an added length effect (i.e., $\varepsilon_{e}$ ) to the neck's depth, is used to account for the mass loading associated with the sound radiation of the neck and to the distortion of the acoustic flow at the resonator surface. In the case of a rigid circular neck opening in contact with two air media on both ends, the two length corrections can be approximated for $s_{e}<0.16$ as proposed by [46]

$$
\varepsilon_{i}=\varepsilon_{e}=0.48 \sqrt{A}\left(1-1.14 \sqrt{s_{e}}\right) .
$$

The resistive part in Eqn. 8 (i.e., $\left.\left(2 d_{n} / R_{n}+4\right) R_{s}\right)$ accounts for the viscous effects occurring within the resonator's neck due to the viscous boundary layer and around its edges at the panel surface due to the distortion of the acoustic flow.

\section{Acoustic properties of $O C$}

Using the transfer matrix of Eqn. 3, several acoustic characteristics of the OC can be determined. Furthermore, Eqns. 3, 5, and 7 represent an acoustic wave impinging on the neck side of the HR (i.e., position AB). Therefore, to obtain the transfer matrix of an $\mathrm{OC}$ placed in the opposite direction (i.e., position BA), Eqn. 3 must be inverted to account for the change in the sign of the velocity [58],

$$
\mathbf{T}^{O C, B A}=\frac{1}{\operatorname{det}\left(\mathbf{T}^{O C, A B}\right)}\left[\begin{array}{ll}
T_{22}^{O C, A B} & T_{12}^{O C, A B} \\
T_{21}^{O C, A B} & T_{11}^{O C, A B}
\end{array}\right] .
$$

The normal incidence sound transmission coefficient $T L_{n}$ is a function of the sound transmission coefficient $\tau: T L_{n}=10 \log _{10}\left(\frac{1}{\tau}\right)$. The transmission factor $\tau$ is the ratio of the power $P_{t}$ transmitted through the acoustic system and the incident power $P_{i}$ : $\tau=\frac{P_{t}}{P_{i}}$. In the case of an anechoic-backed OC, $T L_{n}$ can be obtained by [59]

$$
T L_{n}=-20 \log _{10}\left(\frac{2}{\left|T_{11}^{O C}+T_{12}^{O C} / Z_{0}+Z_{0} T_{21}^{O C}+T_{22}^{O C}\right|}\right) .
$$


The sound absorption coefficient $\alpha$ is a function of the reflection coefficient $R^{b}: \alpha=$ $1-\left|R^{b}\right|^{2}$. The latter is the ratio of the pressures created by the ingoing and outgoing waves at the surface of the acoustic system. The normal incidence reflection coefficient of an OC backed by a rigid back wall, $R^{b}$ can be determined by

$$
R^{b}=\frac{T_{11}^{O C}-Z_{0} T_{21}^{O C}}{T_{11}^{O C}+Z_{0} T_{21}^{O C}} .
$$




\section{Appendix C fabrication}

Two types of origami-based Helmholtz resonators were fabricated:

(i) Rigid 3D printed origami-based resonators: to perform preliminary tests, as well as to better understand the influence of the origami-shaped cavity on the acoustic performances of the device; three rigid origami-based Helmholtz resonators were 3D printed, each with a different folding state: folded, semi-folded and unfolded. The three prototypes were printed from PLA, using an Ultimaker $2 \ddagger 3 \mathrm{D}$ printer.

(ii) Tunable origami-based resonators: to study the effect of tunability on the acoustic properties of origami-based Helmholtz resonators, a tunable resonator was fabricated. The fabrication starts with a patterned, thick sheet of paper. The pattern is obtained either by laser machining or by printing on plain paper, which is then glued to the thick sheet. The sheet is then manually folded and dipped in a flexible glue from Henkel AG Company§. Finally, the neck of the resonator is $3 \mathrm{D}$ printed and glued into its appropriate location. The glue is used to add more stiffness to the cavity of the device, and to ensure that it is correctly closed. As for the thick sheet of paper, it is used as a skeleton to provide the device with its origami shape; also, the thick sheet absorbs the glue better, resulting in an interestingly stiff device.

$\ddagger$ https://ultimaker.com/en/resources/manuals/ultimaker-3d-printers/ultimaker-2

$\S$ https://www.henkel.com/company 
[1] B. H. Lecocq, Contribution à l'étude du comportement acoustique de matériaux présentant des cavites debouchantes, Ph.D. thesis, Ecole Nationale Supérieure d'Arts et Metiers (décembre 2006).

[2] L. Rayleigh, The theory of the Helmholtz resonator, Proceedings of the Royal Society of London. Series A, Containing Papers of a Mathematical and Physical Character 92 (638) (1916) 265-275.

[3] S. M. Kuo, D. R. Morgan, Active noise control: a tutorial review, Proceedings of the IEEE 87 (6) (1999) 943-973.

[4] S. A. Lane, S. Huybrechts, Coupled Helmholtz resonators for acoustic attenuation, Journal of Vibration and Acoustics (2001).

[5] J. S. Anderson, M. Bratos-Anderson, Noise: Its measurement, analysis, rating and control, Gower Technical, 1993.

[6] M. L. Munjal, Acoustics of ducts and mufflers with application to exhaust and ventilation system design, John Wiley \& Sons, 1987.

[7] P. Tang, W. Sirignano, Theory of a generalized Helmholtz resonator, Journal of Sound and Vibration 26 (2) (1973) 247-262.

[8] R. L. Panton, J. M. Miller, Resonant frequencies of cylindrical Helmholtz resonators, The Journal of the Acoustical Society of America 57 (6) (1975) 1533-1535.

[9] R. Chanaud, Effects of geometry on the resonance frequency of Helmholtz resonators, Journal of Sound and Vibration 178 (3) (1994) 337-348.

[10] N. Dickey, A. Selamet, Helmholtz resonators: one-dimensional limit for small cavity length-todiameter ratios, Journal of Sound and Vibration 3 (195) (1996) 512-517.

[11] A. Selamet, I. Lee, Helmholtz resonator with extended neck, The Journal of the Acoustical Society of America 113 (4) (2003) 1975-1985.

[12] S. Tang, On Helmholtz resonators with tapered necks, Journal of Sound and Vibration 279 (3-5) (2005) 1085-1096.

[13] A. Abbad, N. Atalla, M. Ouisse, O. Doutres, Numerical and experimental investigations on the acoustic performances of membraned Helmholtz resonators embedded in a porous matrix, Journal of Sound and Vibration (2019) 114873.

[14] Z. Wang, Y. Choy, Tunable parallel barriers using Helmholtz resonator, Journal of Sound and Vibration 443 (2019) 109-123.

[15] D. Wu, N. Zhang, C. M. Mak, C. Cai, Hybrid noise control using multiple Helmholtz resonator arrays, Applied Acoustics 143 (2019) 31-37.

[16] J. M. De Bedout, Adaptive-passive noise control with self-tuning Helmholtz resonators, Ph.D. thesis, Purdue University (1996).

[17] X. Yu, Z. Lu, F. Cui, L. Cheng, Y. Cui, Tunable acoustic metamaterial with an array of resonators actuated by dielectric elastomer, Extreme Mechanics Letters 12 (2017) 37-40.

[18] A. Abbad, K. Rabenorosoa, M. Ouisse, N. Atalla, Adaptive Helmholtz resonator based on electroactive polymers: modeling, characterization, and control, Smart Materials and Structures 27 (10) (2018) 105029.

[19] A. Benouhiba, K. Rabenorosoa, M. Ouisse, N. Andreff, An origami-based tunable Helmholtz resonator for noise control: Introduction of the concept and preliminary results, in: ASME 2017 Conference on Smart Materials, Adaptive Structures and Intelligent Systems, American Society of Mechanical Engineers Digital Collection, 2017.

[20] C. Zou, R. L. Harne, Adaptive acoustic energy delivery to near and far fields using foldable, tessellated star transducers, Smart Materials and Structures 26 (5) (2017) 055021.

[21] C. Zou, R. L. Harne, Deployable tessellated transducer array for ultrasound focusing and bio-heat generation in a multilayer environment, Ultrasonics (2020) 106108.

[22] T. Cambonie, E. Gourdon, Innovative origami-based solutions for enhanced quarter-wavelength resonators, Journal of Sound and Vibration 434 (2018) 379-403.

[23] S. Babaee, J. T. Overvelde, E. R. Chen, V. Tournat, K. Bertoldi, Reconfigurable origami-inspired acoustic waveguides, Science Advances 2 (11) (2016) e1601019. 
[24] H. Fang, X. Yu, L. Cheng, Reconfigurable origami silencers for tunable and programmable sound attenuation, Smart Materials and Structures 27 (9) (2018) 095007.

[25] Y. Zhu, F. Fei, S. Fan, L. Cao, K. Donda, B. Assouar, Reconfigurable origami-inspired metamaterials for controllable sound manipulation, Physical Review Applied 12 (3) (2019) 034029.

[26] P. P. Pratapa, P. Suryanarayana, G. H. Paulino, Bloch wave framework for structures with nonlocal interactions: Application to the design of origami acoustic metamaterials, Journal of the Mechanics and Physics of Solids 118 (2018) 115-132.

[27] K. Fuchi, A. R. Diaz, E. J. Rothwell, R. O. Ouedraogo, J. Tang, An origami tunable metamaterial, Journal of Applied Physics 111 (8) (2012) 084905.

[28] K. Fuchi, J. Tang, B. Crowgey, A. R. Diaz, E. J. Rothwell, R. O. Ouedraogo, Origami tunable frequency selective surfaces, IEEE antennas and wireless propagation letters 11 (2012) 473-475.

[29] D. M. Sessions, J. T. Ruff, F. A. Espinal, G. H. Huff, S. S. Jape, E. Peraza-Hernandez, D. C. Lagoudas, D. J. Hartl, B. Borges, Folding, tessellation, and deployment of an origami-inspired active-material-enabled self-folding reflector antenna, in: 2018 IEEE International Symposium on Antennas and Propagation \& USNC/URSI National Radio Science Meeting, IEEE, 2018, pp. 929-930.

[30] S. Jape, M. Garza, J. Ruff, F. Espinal, D. Sessions, G. Huff, D. C. Lagoudas, E. A. P. Hernandez, D. J. Hartl, Self-foldable origami reflector antenna enabled by shape memory polymer actuation, Smart Materials and Structures 29 (11) (2020) 115011.

[31] H. v. Helmholtz, Theorie der luftschwingungen in röhren mit offenen enden., Journal für die reine und angewandte Mathematik 57 (1860) 1-72.

[32] J. Rayleigh, The theory of sound,(1945) (1945).

[33] U. Ingard, On the theory and design of acoustic resonators, The Journal of the acoustical society of America 25 (6) (1953) 1037-1061.

[34] M. Alster, Improved calculation of resonant frequencies of Helmholtz resonators, Journal of Sound and Vibration 24 (1) (1972) 63-85.

[35] R. L. Panton, J. M. Miller, Resonant frequencies of cylindrical Helmholtz resonators, The Journal of the Acoustical Society of America 57 (6) (1975) 1533-1535.

[36] S. Fujimoto, M. Nishiwaki, Sojo suru origami asobi no shotai (invitation to creative origami playing), Asahi Culture Center (1982).

[37] B. Kresling, Plant" design": mechanical simulations of growth patterens and bionics, Biomimetics 3 (1996) 105-120.

[38] Y. Chen, H. Feng, J. Ma, R. Peng, Z. You, Symmetric waterbomb origami, Proceedings of the Royal Society A: Mathematical, Physical and Engineering Sciences 472 (2190) (2016) 20150846.

[39] K. Kuribayashi, K. Tsuchiya, Z. You, D. Tomus, M. Umemoto, T. Ito, M. Sasaki, Self-deployable origami stent grafts as a biomedical application of ni-rich tini shape memory alloy foil, Materials Science and Engineering: A 419 (1) (2006) 131-137.

[40] C. D. Onal, R. J. Wood, D. Rus, Towards printable robotics: Origami-inspired planar fabrication of three-dimensional mechanisms, in: Robotics and Automation (ICRA), 2011 IEEE International Conference on, IEEE, 2011, pp. 4608-4613.

[41] D.-Y. Lee, J.-S. Kim, S.-R. Kim, J.-S. Koh, K.-J. Cho, The deformable wheel robot using magicball origami structure, in: ASME 2013 international design engineering technical conferences and computers and information in engineering conference, American Society of Mechanical Engineers, 2013, pp. V06BT07A040-V06BT07A040.

[42] N. Karnessis, G. Burriesci, Uniaxial and buckling mechanical response of auxetic cellular tubes, Smart Materials and Structures 22 (8) (2013) 084008.

[43] S. Chen, R. Karthikeyan, S. C. Ryu, Towards the design of mechanically superior tubular structures for microcatheters, Smart Materials and Structures 28 (3) (2019) 035032.

[44] O. Doutres, N. Atalla, H. Osman, Transfer matrix modeling and experimental validation of cellular porous material with resonant inclusions, The Journal of the Acoustical Society of America 
137 (6) (2015) 3502-3513.

[45] A. E-1050, Standard test method for impedance and absorption of acoustical materials using a tube, two microphones and a digital frequency analysis system (2012).

[46] J. Allard, N. Atalla, Propagation of sound in porous media: modelling sound absorbing materials 2e, John Wiley \& Sons, 2009.

[47] M. R. Stinson, The propagation of plane sound waves in narrow and wide circular tubes, and generalization to uniform tubes of arbitrary cross-sectional shape, The Journal of the Acoustical Society of America 89 (2) (1991) 550-558.

[48] G. Yu, D. Li, L. Cheng, Effect of internal resistance of a Helmholtz resonator on acoustic energy reduction in enclosures, The Journal of the Acoustical Society of America 124 (6) (2008) 35343543.

[49] D. M. Photiadis, The effect of wall elasticity on the properties of a Helmholtz resonator, The Journal of the Acoustical Society of America 90 (2) (1991) 1188-1190.

[50] D. C. Lagoudas, Shape memory alloys: modeling and engineering applications, Springer, 2008.

[51] P. Butaud, M. Ouisse, K. Jaboviste, V. Placet, E. Foltête, Dynamical mechanical thermal analysis of shape-memory polymers, in: Shape Memory Polymers, Blends and Composites, Springer, 2020, pp. 129-151.

[52] J. Mitani, Oripa (origami pattern editor), available at: http://mitani. cs. tsukuba. ac. jp/floripa (2005).

[53] W. Lauriks, P. Mees, J. F. Allard, The acoustic transmission through layered systems, Journal of sound and vibration 155 (1) (1992) 125-132.

[54] K. Verdière, R. Panneton, S. Elkoun, T. Dupont, P. Leclaire, Transfer matrix method applied to the parallel assembly of sound absorbing materials, The Journal of the Acoustical Society of America 134 (6) (2013) 4648-4658.

[55] K. Verdière, R. Panneton, S. Elkoun, T. Dupont, P. Leclaire, Comparison between parallel transfer matrix method and admittance sum method, The Journal of the Acoustical Society of America 136 (2) (2014) EL90-EL95.

[56] A. Pellicier, N. Trompette, A review of analytical methods, based on the wave approach, to compute partitions transmission loss, Applied Acoustics 68 (10) (2007) 1192-1212.

[57] B. Efimtsov, L. Lazarev, Sound transmission loss of panels with resonant elements, Acoustical Physics 47 (3) (2001) 291-296.

[58] R. Panneton, Normal incidence sound transmission loss evaluation by upstream surface impedance measurements, The Journal of the Acoustical Society of America 125 (3) (2009) 1490-1497.

[59] A. International, Astm e2611-09 standard test method for measurement of normal incidence sound transmission of acoustical materials based on the transfer matrix method, ASTM, 2009. 